\title{
Desafíos actuales de la enseñanza de la filosofía
}

\author{
Juan Guillermo Díaz Bernal \\ Óscar Pulido Cortés \\ Editores invitados \\ Universidad Pedagógica y Tecnológica de Colombia (UPTC)
}

pp. $11-18$

La enseñanza de la filosofía se ha venido constituyendo como un campo teórico y metodológico en el último tiempo. Las continuas amenazas que se ciernen sobre este saber milenario, y que proceden de los más variados contextos (políticos, académicos, culturales, etc.), han concitado una defensa especial alrededor de la posibilidad de seguir siendo enseñado en colegios y universidades. Esta defensa se manifiesta no solo en las luchas políticas y normativas que se han dado en diversos países, sino también en la creación de asignaturas, programas, proyectos de investigación, redes de maestros y conceptos. El informe de la UNESCO publicado por primera vez en francés en 2007 y cuyo título en castellano es La Filosofía, una escuela de la libertad: enseñanza de la filosofía y aprendizaje del filosofar; la situación actual y las perspectivas para el futuro (2011) produjo un completo estado de la cuestión sobre la enseñanza de la filosofía a nivel mundial.

Hoy, 12 años después, la situación sigue consolidándose como preocupación, aunque también como esperanza. En el primer caso, porque los gobiernos neoliberales y reaccionarios consideran que la filosofía es algo inútil y peligroso en la formación de las nuevas generaciones. En el segundo caso, porque hay una manifestación vigorosa de prácticas filosóficas contemporáneas que auguran un futuro prometedor para nuestra disciplina. Para el caso colombiano, diversos estudios (Díaz y Espinel, 2019; Pulido, Espinel y Gómez, 2018) han demostrado cómo la reflexión sobre la enseñanza de la filosofía ha venido ganando espacio en publicaciones especializadas de filosofía y educación. Ante este panorama, la revista Cuestiones de Filosofía no pretende ser la excepción y por eso dedica este número a contribuir con la discusión nacional y latinoamericana sobre el particular. 
La filosofía se justifica plenamente en la formación y su presencia se hace absolutamente imprescindible en la enseñanza. Dada esa historicidad radical de nuestro existir, nuestro modo de ser no es una realidad ya terminada, sino un continuo devenir, un proceso en construcción con la necesidad de revisar, constantemente hoy, el concepto de formación. La educación escolar no resuelve todos los problemas de la sociedad colombiana ni tampoco de la formación de las personas. Lo que, efectivamente, podría hacer es contribuir con un esfuerzo de formación sin tener que responder a su integralidad. En el ámbito de la escuela, no cabe una única disciplina como encargada de la formación integral de los educandos. Ninguna disciplina puede hacer eso sola, lo formativo es responsabilidad de todas las disciplinas, del currículo en un sentido amplio, con el contexto y las personas inmersas.

Para educar a las nuevas generaciones, es necesario que el proceso de formación y desarrollo se construya colectivamente. Es necesario tener en cuenta la historia y la solidaridad del proceso humanista de nuestro tiempo. No hay nada mejor que trabajar con las nuevas generaciones en el mundo del trabajo, de la participación social, de la cibercultura, de la automatización y de la libertad; por eso, la función de la locación es exactamente explorar la herramienta del conocimiento. Para la educación, los currículos, la enseñanza, entre otros, pueden tornarse significativos en cualquiera de los niveles de formación humana, modalidades en las cuales es necesario lidiar adecuadamente con los saberes mosaico. Esto quiere decir que no hay un proceso de enseñanza ni de aprendizaje, sino que hay un proceso de producción y construcción de conocimiento. Entonces, ahí aparece la importancia del abordaje filosófico, de la postura filosófica, de la actitud filosófica; no como una determinada función en particular, sino pasando por un proceso de inmersión en la cultura contemporánea.

De cualquier manera, es necesario priorizar aquello que es fundamental: la formación filosófica. Entendiéndose a la filosofía con esta postura, con esta actitud del espíritu humano, en relación con las coordenadas de su propia existencia histórica, social y cultural; por eso, es necesaria en todos los niveles de la enseñanza. Al hablar hoy desde la práctica y la enseñanza de la filosofía, nos remitimos al ejercicio de la subjetividad como lo que nos hace recordar que toda actividad intelectual humana, todo conocimiento con expansión-subjetividad está íntimamente articulado con la existencia del hombre. 


\section{III}

\section{Enseñar filosofía}

Dada la relevancia y el imprescindible ejercicio de la reflexión filosófica en el seno de la cultura, la enseñanza de la filosofía merece un cuidado muy especial, ya que es el locus principal del desencadenamiento de todo proceso en la búsqueda del sentido. No se trata solamente de instruirse en una determinada habilidad ni de apropiarse de un acervo de conocimientos. Se trata, ante todo, de desarrollary madurar un estilo de reflexión, un modo de pensar, una manera especial de subjetividad. Esto puede ser conquistado a través de las mediaciones pedagógicas que son imprescindibles a la hora de enseñar filosofía. De hecho, una de las tendencias contemporáneas tiene como fundamento mirar a la enseñanza de la filosofía como una particular puesta en escena a partir de la concepción que de ella tienen los maestros y de las relaciones que se pueden establecer entre los textos, los interlocutores y los productos del pensamiento.

\section{Educación filosófica}

La relación entre filosofia e infancia es determinante, dado que posibilita en los niños y niñas el ejercicio de un diálogo filosófico que tiene incidencia en el desarrollo de la capacidad de pensar sistemáticamente. Al reiterar la pertinencia de los esfuerzos que en este sentido se vienen haciendo, con la intención de buscar filosóficamente la relación entre el proceso de formación y desarrollo de los estudiantes en todos los momentos de su tiempo escolar, se reconoce que la procedencia de la experiencia pedagógica se halla ligada a la actividad filosófica desde la básica primaria. Eso sin dejar de desconocer todas las cautelas y las eventuales limitaciones padecidas hasta el dia de hoy.

Igualmente válidas son la comunidad de indagación y la expansión de la formación filosófica de los adolescentes en el bachillerato o en la básica secundaria y media. Indiscutiblemente, su relevancia en esta fase resulta determinante en la formación de la identidad del ser humano. Así, la formación filosófica de niños y adolescentes es relevante, una vez que esa formación contribuye a los campos prácticos de la filosofia. Aquí se visibilizan las prácticas filosóficas contemporáneas como el café filosófico, la asesoría filosófica y, en especial, el programa de filosofía para y con niños, que en Colombia se ha consolidado luego de la década del 2000 (Suárez, Lara y González, 2017; Cañizales y Pulido, 2015; Morales y Pulido, 2018). 


\section{Aprender filosofía}

El ejercicio del filosofar implica un diálogo especial con los pensadores del pasado $y$, a su vez, con los contemporáneos. Tanto en un caso como en el otro, no estamos delante de un producto sui generis del cual nos apropiamos para una especie de proceso de pensamiento, reflexión, indagación o, quizás, una búsqueda de sentido de nuestra experiencia o existencia. Por el contrario, el proceso de aprender filosofía se articula a las diferentes prácticas filosóficas que generan un debate original. Aprender a pensar-pensando justifica la significación más profunda del diálogo con los pensadores que nos antecedieron en el tiempo y con aquellos que conviven con nosotros en la esfera pública. Así, la práctica filosófica se hace paideía, es decir, pedagogía para formar al ciudadano; por eso, se necesita de lo real. Formar a la juventud y formar a los formadores, a los educadores en general y a los filósofoseducadores en particular, es habilitarlos en el ejercicio de una forma de pensamiento que sea creativa y crítica con relación a la realidad del existir (Espinel y Pulido, 2017).

\section{Didáctica de la filosofía}

Los textos clásicos de la filosofía constituyen la base para la reflexión, la disertación y el debate filosófico como conclusión a un proceso de formación. Los textos filosóficos tienen una incuestionable dimensión didáctica. El contacto y la convivencia con los textos impiden que se caiga en la mera opinión del sentido común o en la levedad del consumo. El trabajo del concepto es un esfuerzo necesario para que no predomine en el espíritu de los iniciantes en filosofía un cierto sentimentalismo, tendencia siempre presente cuando se pretenden debatir cuestiones que tienen que ver con las condiciones de la existencia de los seres humanos. El abordaje didáctico de la filosofía se apoya en el objetivo de la formación tal y como actúa el filósofo, es decir, en su práctica de reflexión con los temas y problemas generales relacionados con la existencia humana y mediados por los temas específicos de la experiencia vivida en los diversos ámbitos de nuestro existir. 


\section{IV}

Ahora bien, este número de Cuestiones de Filosofía tiene como propósito presentar trabajos de investigación que discuten desde diferentes aristas las relaciones enseñanza-filosofía y filosofía-educación. En esta oportunidad, la revista se compone de siete trabajos de reconocidos especialistas en las áreas que son objeto de interés. El primero de ellos se titula "Una propuesta de orientaciones pedagógicas desde la filosofía de Nuestramérica para la enseñanza de la filosofía en Colombia", de los profesores Andrés Carmona Cardona (Institución Educativa Josefina Muñoz González) y Diana Melisa Paredes Oviedo (Universidad de Antioquia). El objetivo de los autores estriba en proponer una revisión de los cambios ocurridos a partir de la década del noventa, en el que se pasa de una enseñanza de la filosofía centrada en los contenidos y procesos histórico-conceptuales a su especificidad en el desarrollo de competencias, lo que lleva a la fusión de la filosofía con la llamada "lectura crítica" en las pruebas o exámenes de Estado. La propuesta de Carmona Cardona y Paredes Oviedo se ampara en una teoría decolonial y crítica que sirve de piedra de toque para resignificar el concepto de formación filosófica situada y para reelaborar el concepto de competencia en perspectiva de desarrollo humano.

El segundo texto es del reputado investigador Armando Zambrano Leal (Secretaria de Educación del Valle del Cauca) y lleva por título "Séneca, pedagogo: Cuidado y acompañamiento en las cartas a Lucilio". En este sugestivo artículo, Zambrano Leal problematiza al pedagogo en el sitio del acompañamiento y del cuidado del otro, más que en la instrucción, la cual ha sido privilegiada en las acciones educativas del mundo moderno. De igual manera, el autor discute el concepto de formación: en especial, el de formación del pedagogo, que ha sido blanco tanto de la racionalidad técnica como de los dispositivos de aprendizaje.

En tercer lugar aparece un artículo de tres profesoras de la Universidad Nacional del Sur (Argentina). Con "Enseñanza filosofante. Una perspectiva transescolar", Maria Belén Bedetti, Laura Susana Morales y Maria Laura Medina abordan un concepto novedoso, a saber: la filosofía en perspectiva transescolar. Con el susodicho, las profesoras argentinas aportan elementos de juicio que posibilitan la superación de la vieja discusión entre enseñar filosofía o enseñar a filosofar, y que permiten ubicar 
a nuestra disciplina en espacios diferenciados y a la vez conectados con prácticas contemporáneas como la filosofía para niños, los cafés filosóficos y la filosofía de indole académica y universitaria.

"La educación es una técnica de domesticación: una perspectiva para pensar las prácticas educativas contemporáneas" figura como cuarto trabajo. Aqui el profesor Julio Andrés Cifuentes Chauta (Colegio Fernando Soto Aparicio), a partir del concepto de antropotécnica de Sloterdijk, reflexiona acerca de la hipótesis moderna de la educación como un proceso de domesticación que hace que el hombre vuelva sobre si mismo y pueda realizar una vida mejor tanto en lo individual como en lo colectivo. A juicio de Cifuentes Chauta, la educación como domesticación se convierte en el proceso de formación que privilegia el hábito y la ejercitación como bases de la educación humanizante.

Un quinto texto es el del profesor mexicano Alejandro Roberto Alba Meraz, vinculado a la Universidad Nacional Autónoma de México (UNAM). En "Hacia una concepción experimental de la filosofía en la escuela", el autor reflexiona en torno al sitio que ocupa la filosofía en el currículo escolar a partir de las reformas contemporáneas. La propuesta de trabajo busca volver a promover la reflexión filosófica en la escuela como forma de desaprender en el periodo escolar, esto es, como forma de sensibilizar a estudiantes y docentes para poder ver el mundo de otras posibilidades diferentes a las hegemónicas.

Los dos últimos artículos vienen de dos universidades brasileñas: de la Universidade Federal do Rio de Janeiro (UFRJ) y de la Universidade Federal de Uberlândia (UFU). El profesor Filipe Ceppas, de la UFRJ, es el autor de "Ensino de filosofia na encruzilhada: democracia, atualidade e antropofagia"; entre tanto, los profesores Márcio Danelon y Mauro Sérgio Santos da Silva, ambos de la UFU, escriben "Planejamento do ensino de Filosofia: entre a normatividade e a prática pedagógica". El objetivo de Ceppas, basado en el proyecto antropofágico de Oswald de Andrade, es derribar los límites de una formación escolar y filosófica europea. Para ello, se vale de algunos conceptos de Walter Benjamin y de las posibles apropiaciones de la herencia indigena latinoamericana. En este sentido, resulta pertinente la indagación por la función de la enseñanza de la filosofía y de la formación para la ciudadanía en el 
Brasil contemporáneo. Por su parte, Danelon y Santos da Silva buscan poner de manifiesto el exceso de documentos que regulan la enseñanza de la filosofía en Minas Gerais. Su pesquisa se basa en el análisis exhaustivo de las políticas públicas para la enseñanza de la filosofía y las prácticas de los maestros de filosofía en las escuelas secundarias de esa región brasileña.

Finalmente, los lectores podrán encontrar una entrevista que la profesora Liliana Andrea Mariño Díaz le realiza a Diego Antonio Pineda Rivera, quien a la sazón es el estudioso colombiano más importante del programa Filosofía para Niños (FpN). En la entrevista, el profesor Pineda Rivera desarrolla la categoria de educación filosófica y sus implicaciones para la filosofía y la educación en Colombia.

Como editores, queridos lectores, los invitamos a disfrutar de cada uno de los textos de esta nueva entrega de Cuestiones de Filosofía. Sin lugar a dudas, las reflexiones que nos aportan los prestigiosos autores servirán para esclarecer y fomentar el debate en torno a las relaciones entre enseñanza-filosofía y filosofía-educación.

\section{Referencias}

Cañizalez, N., y Pulido, O. (2015). Infancia, una experiencia filosófica en el cine. Praxis \& Saber, 6(11), pp. 245-262.

Diaz, J., y Espinel, O. (Eds.). (2019). Fragmentos. Leer, traducir, dialogar. Bogotá: Editorial Unimiunto.

Espinel, O., y Pulido, O. (2017). Enseñanza de la filosofía. Entre experiencia filosófica y ensayo. Universitas Philosophica, 34(69), pp. 121-142.

Mariño, L., Pulido, O., y Morales, L. (2016). Actitud filosófica, infancia y formación de maestros. Praxis \& Saber, 7(15), pp. 81-101.

Morales, L., y Pulido, O. (2019). Ambientes filosóficos para la lectura en la escuela rural. Praxis \& Saber, 9(21), pp. 99-124. 
Pulido, O., Espinel, O., y Gómez, M. (Eds.). (2018). Filosofía y enseñanza. Miradas en Iberoamérica. Tunja: Editorial UPTC.

Suárez, M., González, B., y Lara, P. (2017). Apropiaciones y experiencias pedagógicas de filosofia e infancia en Colombia. Praxis \& Saber, 8(16), pp. 225-247.

UNESCO. (2011). La Filosofía, una escuela de la libertad: enseñanza de la filosofía y aprendizaje del filosofar; la situación actual y las perspectivas para el futuro. México: UNESCO. 